\title{
Taming Uncertainty
}

Ralph Hertwig, Timothy J. Pleskac, Thorsten Pachur, and The Center for Adaptive Rationality 


\title{
1 Reckoning with Uncertainty: Our Program of Research
}

\author{
Ralph Hertwig, Timothy J. Pleskac, and Thorsten Pachur
}

\subsection{Uncertainty as the Human Condition}

At a press conference held in East Berlin on the evening of November 9, 1989, Günter Schabowski, a spokesman for the socialist regime of the German Democratic Republic (GDR), read out what seemed like a secondary item on a list of mundane announcements:

A decision was made today, as far as I know.... A recommendation from the Politburo was taken up that we take a passage from [a draft of] the travel regulation and put it into effect, that, (um) - as it is called, for better or worse-that regulates permanent exit, leaving the Republic. Since we find it (um) unacceptable that this movement is taking place (um) across the territory of an allied state, (um) which is not an easy burden for that country to bear. Therefore (um), we have decided today (um) to implement a regulation that allows every citizen of the German Democratic Republic (um) to (um) leave the GDR through any of the border crossings. (Hertle, 2001, p. 157)

Schabowski continued on, but was interrupted by a reporter with a question that would change the world: "When does it come into effect?" Visibly uncertain, mumbling to his aides and thumbing through his papers, he finally said, "to my knowledge ... immediately, without delay" (Hertle, 2001, p. 158). It is unlikely that he could have imagined the chain of events that these words would set off. Suddenly, the people of the GDR were thrust into their own state of uncertainty: Would the country's armed border guards obey the new travel regulations, granting complete freedom to travel, or would they use force to prevent any border crossing? Would the country's leaders revoke the announcement once its effects became clear, choosing to shed the blood of their people rather than risk a hemorrhaging of their population? Within hours, as joy vied with fear, tens of thousands of East Germans converged on the crossing points to West Berlin chanting "Tor 
auf!" "Open the gate!"). Stunned, outnumbered, and lacking any information on the new policy or orders from the military leadership, the border guards ad-libbed-and opened the crossings. This division at the heart of Europe, which was literally set in stone, vanished overnight-and with it the certitudes, plans, and projections of millions of people.

Predictions about repercussions varied wildly. The GDR leadership had hoped the travel law would strengthen its regime (Hertle, 2001; Meyer, 2015). French President François Mitterrand, in contrast, believed that the Soviet leadership would never accept this development and that the Germans were unwittingly risking a world war. The very next day, Soviet leader Mikhail Gorbachev warned the leaders of France, the United Kingdom, and the United States of a possible "destabilization of the situation not only in the center of Europe but also beyond" (quoted in Hertle, 2001, p. 138). None of these things came to pass. As the saying often attributed to physicist Niels Bohr goes: Prediction is very difficult, especially about the future.

The quip highlights three more general properties of the human struggle with uncertainty. First, much of what people do-not only in politics but also in ordinary life-is predicated on forecasts of the future. Whether choosing a job, an apartment, or a spouse; whether deciding when to travel the world, have children, or start saving for old age; whether voting in an election or deciding between medical treatments, people base their decisions on predictions about what the future holds. Second, prediction can be difficult because most future events are shrouded in uncertainty-indeed, virtually all the political players in the fall of the Berlin Wall got it wrong (Hertle, 2001). Third, uncertainty and lack of knowledge bedevil not only people's predictions of the future but also their mental constructions of the present and reconstructions of the past (Loftus, 1993; Schacter, 1999; S. M. Stigler, 1980). "Ask any American who brought down the Berlin Wall, and nine of 10 will say Ronald Reagan," said former Secretary of State James Baker, but "we had hardly anything to do with it" (Meyer, 2015, para. 14).

\subsection{Our Program of Research: Understanding the Adaptive Toolbox for Taming Uncertainty}

If people inhabit "isolated islands of certainty in an ocean of uncertainty" (Arendt, 1958/2013, p. 244), this book is about how people navigate the high seas. Even under daunting conditions-where knowledge is imperfect, 
complexity is high, and time is short-people make predictions, inferences, and decisions so close to effortlessly that they have been called "masters of prediction" (Clark, 2016), and the accuracy of many of their predictions excels that of the political players in the fall of the Berlin Wall. What are the foundations of this mastery? In this book, we argue that they are a set of tools that the human mind-as an evolved and continuously learning cognitive system-has developed to grapple with uncertainty. And just as a good mechanic has multiple tools, each designed for a specific purpose, the human cognitive system has specific tools for dealing with the different forms of uncertainty it encounters (see chapter 18; Lo \& Mueller, 2010).

With this notion of cognition, we challenge the idea that people manage uncertainty as if they were reducing it to risks, that is, as if they could summon at least subjective numerical probabilities for the outcomes of every decision they make (originally proposed by Savage, 1954). If they could, probability would constitute the very currency of human thought. Instead, we argue that people master the myriad types of uncertainty they face, from the inherent unpredictability of their environment (environmental uncertainty) to their limited knowledge and understanding of other people's actions and intentions (strategic uncertainty), by deploying a wide range of cognitive tools, many of which have no or only a rudimentary need to know the probabilities, let alone the utilities, of outcomes. We refer to this repertoire of tools for making predictions, inferences, and decisions as the mind's adaptive toolbox.

Guided by this concept, we build on our own and others' work on cognitive tools known as simple heuristics (Gigerenzer, Hertwig, \& Pachur, 2011; Gigerenzer, Todd, \& the ABC Research Group, 1999; Hertwig \& Hoffrage, 2013; J. W. Payne, Bettman, \& Johnson, 1993; Todd \& Gigerenzer, 2012). Inspired by Herbert Simon and his vision of bounded rationality $(1955,1982$, 1990), this work has proposed that much of human (and animal) reasoning, decision making, and behavior can be modeled in terms of such heuristics, which rest on simple principles of information processing and often consider only a subset of the information available. Heuristics are a realistic alternative to more classical approaches to decision making under uncertainty, such as expected utility theory and subjective expected utility theory, which often rest on extravagant implicit requirements and assumptions about the information, processing capacity, and time available (see Simon, 1955, pp. 103104; see also chapter 2). 
Simple heuristics constitute one important class of cognitive tools for reckoning with uncertainty. But a good mechanic's toolbox holds many handy implements in its drawers and trays: calipers for taking measurements; a flashlight to search for information and diagnose the problem so as to select the right tool; wrenches, ratchets, and pliers to fix it. And just as a mechanic can consult with colleagues and friends on tricky problems, people facing uncertainty can deploy social tools. Acknowledging the many facets of the adaptive toolbox, our research program examines two other important sets of cognitive tools, both of which serve to reduce reducible uncertainty or deal with irreducible uncertainty. One set supports search, permitting people to glean information about the future by learning from the environments they encounter. The second set has people teaming up with others and tapping their collective intelligence, that is, a group's ability to sometimes outperform individual decision makers when solving cognitive problems.

Our first goal in this book is to train the spotlight on these three sets of cognitive tools-heuristics, search strategies, and crowd aggregation ruleswhich we consider indispensable for reckoning with uncertainty. A second goal is to reveal the dynamic nature of the adaptive toolbox. Cognitive tools develop in response to changes inside the mind-for example, when cognitive resources like memory or knowledge grow or decline, or preferences concerning risk change. They also respond to changes outside the mind, such as when environmental demands shift (e.g., from exploring the world and forming alliances to finding a partner and raising children). Such developmental changes influence how the mind reckons with uncertainty and what kind of uncertainty it faces. How cognitive tools such as heuristics or search strategies are selected generally depends on factors such as the person's amount of accumulated knowledge (e.g., what they do and do not recognize), working memory capacity, value processing, and cognitive control, each of which has a distinct developmental trajectory. To understand how the mind handles uncertainty, it is therefore also imperative to understand how the adaptive toolbox is impacted by and develops along with the mind using it, as this book's handful of examples of the toolbox's mutability over the course of developmental change within individuals (ontogenetic change) and across generations of individuals (phylogenetic change) make clear.

Our third goal pertains to the kind of rationality that can arise from the relationship between the adaptive toolbox and the environment. Any 
cognitive tool will work in some contexts but not in others: its rationality is domain-specific rather than general. Like a lock and key (Barrett, 2005; see also Barrett \& Kurzban, 2006), both the architecture of the cognitive tool and the respective environment must be investigated to determine how well they fit together. In this book, we extend this ecological rationality approach beyond the study of heuristics (e.g., Arkes, Gigerenzer, \& Hertwig, 2016; Todd, Gigerenzer, \& the ABC Research Group, 2012) to search strategies and crowd aggregation rules as well. Like heuristics, search strategies will succeed in some environments but fail in others; the same applies to strategies that harness collective intelligence. The ecological rationality of all these cognitive tools means that there is no master key. The lack of a master key, in turn, implies there are notable costs to using an adaptive toolbox, since the tools that empower the mind to deal with uncertainty are themselves a source of uncertainty. When should a specific tool be deployed? In this book, we seek to identify where tools and environments fit together, and where they do not; in so doing, we advocate a systemic view of uncertainty. This approach locates uncertainty neither solely in the mind (epistemic uncertainty) nor solely in the environment (aleatory uncertainty) but highlights the interactive dynamic of the two (see also chapter 18).

In summary, this book aims to advance the understanding of how the mind deals with uncertainty on three fronts. First, we integrate three important dimensions of human decision making into the concept of the adaptive toolbox: (a) boundedly and ecologically rational heuristics, (b) cognition as a search process, and (c) the tools the mind uses to tap into collective intelligence. Each dimension represents influential visions of human (and animal) cognition that have previously existed apart. Second, we advocate the view that the mind's repertoire of cognitive tools is anything but staticnot only the toolbox but also its cognitive foundation and the environment are in constant flux and subject to developmental change. Finally, we demonstrate that each cognitive tool can be analyzed by enlisting the concept of ecological rationality, that is, the fit between specific tools and specific environments. All three goals are informed by the desire to further the understanding of what Arrow (1951) called "realistic" (p. 404) theories of how people make decisions without complete knowledge and, it should be added, where time and computational capacities are limited.

In seeking to capture how real people, alone and in tandem with others, make decisions under uncertainty, we take a decidedly different path 
than the ones trod by others. Many scholars of the mind see cognition in an uncertain world in terms of a single universal, computationally powerful and optimizing prediction machine-whether Bayesian in nature (Clark, 2016; Griffiths, Chater, Kemp, Perfors, \& Tenenbaum, 2010; but see Jones \& Love, 2011), resting on neural networks (McClelland et al., 2010; Rumelhart, McClelland, \& the PDP Research Group, 1986), or involving a combination of both (e.g., Bayesian deep learning). Others leave the well-worn path of assuming classical information processing properties in favor of alternatives such as quantum information processing (Busemeyer $\&$ Bruza, 2012). Each approach has merits and has yielded new insights. For instance, it is useful to understand how the mind might go about implementing an optimal solution (Gershman, Horvitz, \& Tenenbaum, 2015), or to what extent the principles of quantum information processing shed light on certain psychological regularities (Kvam, Pleskac, Yu, \& Busemeyer, 2015). The adaptive toolbox approach we offer here seeks to understand the mind's amazing machinery for prediction, inference, and decision making as a repertoire of psychologically realistic strategies that fit specific environments. This toolbox promises flexibility and efficiency, thus equipping cognition with the ability to unlock the information that ever-changing environments carry about the uncertain future. We now turn to the conceptual issues that guide our investigations in the four major parts of this book: the heuristic mind, the exploring mind, the social mind, and the unfinished mind.

\subsection{The Heuristic Mind}

Heuristics are one of the most important types of realistic decision-making tools for coping with uncertainty. Each heuristic's policy represents a wager on the structure of the environment in question; it bets that ignoring some of the (often noisy) available information will enable faster, and potentially even more accurate, decisions (Gigerenzer \& Gaissmaier, 2011). Indeed, one of the major discoveries of research on simple heuristics is that they are sometimes more accurate than complex procedures (e.g., Gigerenzer \& Goldstein, 1996; Hertwig \& Todd, 2003; Pachur, 2010; Pleskac, 2007). This discovery challenges the standard explanation of people's reliance on heuristics, which frames it as a kind of compromise between minimizing cognitive effort and maximizing accuracy (Shah \& Oppenheimer, 2008). From 
this perspective, people rely on heuristics because searching for and processing information is taxing. Heuristics offer relief by trading accuracy for faster and more frugal cognition. This accuracy-effort trade-off-which is sometimes conceptualized as rational (J. W. Payne et al., 1993) and at other times as seriously flawed (leading to "cognitive illusions"; see Gilovich, Griffin, \& Kahneman, 2002; Kahneman, Slovic, \& Tversky, 1982)-seems to have been accepted as a potentially universal law of cognition.

The world's complexity is unquestionably beyond the grasp of the individual mind, but this David-versus-Goliath imbalance may not be why people rely on heuristics. The fact that simple heuristics can outperform more complex strategies raises a different possibility: the use of heuristics may reflect the power of heuristics rather than the weakness of the mind. Heuristics can be versatile and competitive under at least two conditions: when they are deployed in appropriate environments-that is, when the degree of tool-to-environment fit is high-and equally important, when information about the decision environment is scarce (e.g., Gigerenzer \& Brighton, 2009; Katsikopoulos, Schooler, \& Hertwig, 2010). This second condition is likely to be the rule rather than the exception in the complex environments that humans inhabit.

Brunswik (1957/2001) likened cognition and the environment to a married couple who have to come to terms with each other through mutual adaptation. The concept of ecological rationality (Todd, Gigerenzer, et al., 2012) highlights the fit between a heuristic - or, as we propose in this book, of any decision-making tool-and an environment. It also raises the following questions:

What structures does the environment offer that a decision-making tool could exploit?

In which environments does a particular decision-making tool succeed?

Which decision-making tools succeed in a particular environment?

How do decision-making tools and environments coevolve?

Cognitive science, psychology, and behavioral economics have often been content to study just one half of the couple: the mind's "software" and capacities. But as any marriage counselor knows, listening to just one side of the story will probably not illuminate why and when a marriage does or does not work. The same holds for cognitive strategies. In research on ecologically rational heuristics, considerable progress has been made toward 
understanding the intersection between the environment and the mind (Fawcett et al, 2014; Hogarth \& Karelaia, 2006; Martignon \& Hoffrage, 1999, 2002; Pachur, Hertwig, \& Rieskamp, 2013a; Pleskac \& Hertwig, 2014; Şimșek, 2001; Şimşek \& Buckmann, 2015; Todd, Gigerenzer, et al., 2012). Yet the study of the interplay between the mind and the environment is still in its infancy; important challenges remain. In the second part of this book, titled "The Heuristic Mind," we take on three of these challenges.

\subsubsection{How Do Preferential Choice Heuristics Handle Uncertainty?}

From the outset, research on ecologically rational heuristics and "Homo heuristicus" (Gigerenzer et al., 2011) has focused on the domain of inference (e.g., which of these paintings is more valuable?) rather than preference (e.g., which of these two paintings would you like to have?). The key reason is that the domain of inference involves external criteria (e.g., the monetary value of a painting) and, therefore, commonly accepted benchmarks for evaluating the performance of inferential heuristics and people's use of them. Since benchmarks for preference are less clear, "accuracy" of choice in this context has been defined in many ways, ranging from adherence to coherence criteria (e.g., transitivity; see Pleskac, Diederich, \& Wallsten, 2015) to "gold standards" such as expected value or utility maximization. Examination of choice heuristics in the domain of preference has been limited to the world of risk, where, according to Luce and Raiffa (1957), the outcomes of actions and the probabilities of those outcomes are known (e.g., Brandstätter, Gigerenzer, \& Hertwig, 2006; J. W. Payne et al., 1993). In addition, choice heuristics have typically been invoked to explain systematic violations of, for instance, transitivity and axioms such as independence (Katsikopoulos \& Gigerenzer, 2008; Tversky, 1969, 1972). But what happens when choice heuristics and strategies of rational choice (e.g., expected value theory) engage in decision making under uncertainty, where each action has a set of possible outcomes whose probabilities are not known (Luce \& Raiffa, 1957)? Will some heuristics be on a par with, or even more accurate than, computationally more complex strategies in the domain of preference, where the "Olympian" models of rationality (Simon, 1983, p. 19) were originally proposed (e.g., subjective expected utility theory; Savage, 1954)? If so, in which environments can different heuristics be expected to succeed or fail? Chapter 2 addresses these questions. 


\subsubsection{Which Environmental Structures Are There to Be Exploited?}

The second challenge we take on focuses on the environmental half of Brunswik's (1957/2001) married couple. What structures does the environment offer that might enable ecologically rational decisions? In the domain of inference, for instance, recognition has been identified as an important predictive indicator. It builds on the environmental regularity that objects scoring high on a criterion (such as large cities, wealthy people, or successful athletes) are seen and talked about more frequently than objects that score low. This ecological structure is exploited by the recognition heuristic (D. G. Goldstein \& Gigerenzer, 2002), which interprets the failure to recognize one object and the recognition of another as a sign that the latter scores higher on a given criterion. But there is also an environmental regularity that binds extreme values and frequency differently: the relationship between risk and reward. Chapter 3 focuses on this structure, which exists in many environments. For instance, the bigger the jackpot one can win in a lottery or casino, the smaller the chances of actually winning it. Being cognizant of this regularity is an ecologically smart way to estimate unknown probabilities, thus reducing uncertainty. Chapter 4 demonstrates that structures in the social world (see also chapter 14), such as spatial clustering of social phenomena and people's hierarchical social network structure, can also be exploited to make accurate and frugal inferences about social statistics such as the frequencies of health hazards in the population.

Like Brunswik (1957/2001), Simon (1990) emphasized the collaboration between cognition and environment and insisted that "to describe, predict and explain the behavior of a system of bounded rationality, we must both construct a theory of the system's processes and describe the environments to which it is adapting" (pp. 6-7; emphasis added). The science of bounded rationality has proceeded along these lines by surveying and cataloging choice environments and asking which strategies and solutions would be most effective in each (Marewski \& Schooler, 2011; Şimşek \& Buckmann, 2015; Todd, Gigerenzer, et al., 2012). One downside to this approach is that one may end up with a different heuristic or set of heuristics for each discernable environment or environmental structure, resulting in a multitude of descriptions of environment-heuristic associations. In order to avoid such "description inflation," let us reframe Simon's goal as follows: In order to explain the behavior of a system of bounded 
rationality, we must eventually also construct theories of the system's processes as well as theories of the mechanisms underlying the emergence of classes of environmental structures. Admittedly, this goal is extremely ambitious, but we have already made modest progress toward it. In chapter 3 , we outline a theory that explains and predicts when and why risk-reward structures emerge and, by extension, where a heuristic exploiting this structure can be expected to work well or falter (see Pleskac, Conradt, Leuker, \& Hertwig, 2018).

\subsubsection{Can Heuristics Succeed under Strategic Complexity?}

A third challenge we address is whether the success of heuristics is restricted to static environments that do not require sophisticated strategic responses. There is a firm belief that simple heuristics are destined to fail when employed in "interactions with other intelligent agents, especially competitive agents" (Sterelny, 2003, p. 53). The rationale behind this belief is that social environments populated with other, competitive, agents are much more complex than physical environments (see Hertwig, Hoffrage, \& the $A B C$ Research Group, 2013). In competitive environments, strategies face counterstrategies, ostensibly requiring individuals to proactively interpret and forecast the behavior of others. In this view, individuals need to be aware that others will try to get the better of them, whereas nature, in its dispassionate amorality, will not. Is there any way that heuristics can prevail in these competitive interactions, which seem to require that an individual generate a model of the opponents' behavior, as well as a model of the opponents' model of the individual's behavior, and so on? Chapter 5 gives an answer: Heuristics also hold up in worlds invoking strategic interactions (see also Hertwig \& Herzog, 2009).

To conclude, perhaps the most important discovery in research on simple heuristics is that they can be as accurate as, and sometimes even more accurate than, strategies that make the greatest possible use of information and computation. We address two important challenges to the generality of this finding: In preferential choice, it appears as if heuristics cannot escape an accuracy-effort trade-off; in strategic interactions with competitive agents, it is commonly assumed that heuristics will crash and burn. We also show that heuristics are not just vehicles for describing how people reckon with uncertainty; they also can be explicitly designed and enlisted to help people make better decisions. Chapter 6 analyzes the many uncertainties 
of the modern food environment, which cannot easily be reduced to the stock dimensions of outcomes and probabilities, and illustrates how people can use heuristics to safely navigate a world of carefully crafted temptations (see also chapter 14). But heuristics are just one set of indispensable tools for coping with uncertainty; there is another class of cognitive strategy that is equally important. Adaptive cognition is first and foremost about the smart search for information-the focus of the third part of this book, "The Exploring Mind."

\subsection{The Exploring Mind}

September 30, 1659. I, poor, miserable Robinson Crusoe, being shipwrecked, during a dreadful storm in the offing, came on shore on this dismal unfortunate island, which I called 'the Island of Despair,' all of the ship's company being drowned, and myself dead.

-Daniel Defoe, Robinson Crusoe

Can there be a lonelier and more uncertain world than the one described by Robinson Crusoe? What should he expect? Is the island inhabited? Are the locals friendly or hostile? Should he be worried about wild animals? What food sources are there? Is there drinking water? Where can he find shelter? Will anybody come to his rescue? To cope with these and other existential uncertainties, Crusoe recruited what is perhaps the quintessential tool for making decisions: he explored the island to ascertain the lay of the land, his options, and their possible consequences. In fact, search for informationeither within the bounds of one's mind or in the external world-is what much of cognition is about.

At least two variants of search strategies can be used to reduce a knowledge gap. One is to simulate and forecast the consequences of one's actions on the basis of past data stored in memory-one's own or others' experiences in similar circumstances (e.g., Dudai \& Carruthers, 2005). Of course, relying on past experience to anticipate the future only works if relevant past data are available. Sometimes there are none, as in the case of Crusoe, and sometimes, for whatever reason, the past is a poor predictor of the present or future. In such cases, another search strategy is required, one that permits the individual to explore the world, thus acquiring novel data before deciding. Because the process of sampling information is limited by the decision 
maker's time and capacity, unbounded search is not an option. But limited search does not necessarily imply poor decision making. On the contrary, mirroring the surprising accuracy of simple heuristics, limited search can yield surprisingly good results-whether it targets the information needed for heuristic inference (e.g., Katsikopoulos et al., 2010; Pachur et al., 2013a) or the properties of options in the context of preferential choice (e.g., Hertwig \& Pleskac, 2010; Vul, Goodman, Griffiths, \& Tenenbaum, 2014).

Many normative and descriptive theories of choice, including expected utility theory, subjective expected utility theory, and prospect theory (Kahneman \& Tversky, 1979; Wakker, 2010), are mute on how people search for and learn from information. This reticence might be taken to suggest that how people search contributes little to comprehending how they handle uncertainty. But nothing could be further from the truth. Like Robinson Crusoe, people survive and even thrive in the ocean of uncertainty by enlisting search processes in external and internal environments: visually searching for targets of interest, looking up information on the Internet, or searching their semantic memory (Hills, Jones, \& Todd, 2012). Unless decision scientists comprehend cognition as a search process, they will fail to understand important aspects of human behavior. Consider, by way of illustration, research on risky choice. For at least five decades (E. U. Weber, Shafir, \& Blais, 2004), the field has predominantly studied how people make risky choices by asking them to choose between monetary gambles such as the following:

(A) An $80 \%$ chance of winning $\$ 4$, otherwise nothing, and

(B) $\$ 3$ for sure.

Although the expected value of option $A$ is higher, most people prefer option B. Using choice problems like this, researchers have proposed elegant theories of risky choice that postulate, for instance, how people subjectively represent the objective information given (e.g., subjective functions of probability and outcome information). Yet the process of search is completely missing from these subjective representations and choices; all the necessary information is handed to decision makers on a silver platter. They are thus making decisions from description, in what Edwards (1962a) described as "static" decision tasks where, as Busemeyer (1982) highlighted, there is no need to learn from the past: "When a static decision task is used, the decision maker does not have to learn from past experience with the outcomes 
of previous decisions... This feature of the static decision task becomes a problem when generalizing results to the many day-to-day decisions that repeatedly confront individuals, since explicit information concerning outcome probabilities is frequently not available and must be learned from previous experience" (p. 176).

Indeed, in everyday life, "it is hard to think of an important natural decision for which probabilities are objectively known" (Camerer \& Weber, 1992, p. 325). When people decide whether to start a business or ask someone out on a date, there are no actuarial risk tables to consult. Only by considering uncertainty, the opportunity to search, and the process of stopping search in the choice situation can researchers begin to predict and explain how people arrive at decisions from experience (Hertwig, Barron, Weber, \& Erev, 2004). We and others have studied decisions from experience using the same kind of monetary gambles commonly employed in studies of decisions from description. Systematic comparison of decisions from description with decisions from experience reveals that choices can diverge systematically, an empirical regularity described as the descriptionexperience gap (Hertwig \& Erev, 2009). Chapters 7 and 8 examine this gapwhich could be called a risk-uncertainty gap-and its potential causes. What is increasingly clear is that the major theories developed by decision science to understand decisions from description do not readily apply to decisions from experience. One reason, though not the only one, is that these theories pay no attention to search, the key process through which people pick up on environmental regularities that, in turn, enable them to reduce uncertainty.

\subsubsection{How Search and Choice Are Intertwined}

Search and learning are at the heart of decisions from experience. Therefore, one path forward is obvious: a computational model of a system that explains decisions from experience must specify (a) the processes that guide sampling, (b) the processes that terminate sampling, and (c) the processes that generate a decision on the basis of the sampled information. Models of decisions from experience have attempted to do this in different ways. Often, however, the models treat search and choice as independent processes-and past and new experience as a record or set of records that can be consulted when making a final choice (e.g., Baron, 2005; Fox \& Hadar, 2006; C. Gonzalez \& Dutt, 2011; Hau, Pleskac, Kiefer, \& Hertwig, 2008; Hertwig, Barron, 
Weber, \& Erev, 2006; Plonsky, Teodorescu, \& Erev, 2015). However, much like Brunswik's married couple of cognition and the environment, search and choice are intertwined. How people search shapes the choices they make. For instance, limited search in decisions from experience results in rare events being underrepresented in people's samples; as a result, they make choices as if they were underweighting rare events (see chapters 7 and 8). At the same time, people also choose how to search. They may make a decision once a predetermined level of preference has been reached-in which case, they often choose the last option examined, especially if that option is favorable. It follows that search and choice form a mutually dependent system for making decisions from experience. Chapter 7 lays out the evidence for the codependency of search and choice and proposes a new modeling framework that accounts for this system (see also chapter 4 ).

\subsubsection{The Significance of Search and Learning Extends}

\section{beyond Risky Choice}

Imperfect knowledge and processes of search and learning are not limited to risky choice. In one of the most important tasks the mind has to perform, it must learn which objects belong together. Equipped with limited knowledge of the world's countless objects, the mind often engages actively in search to test classificatory hypotheses (Erickson \& Kruschke, 1998; see also Markant \& Gureckis, 2014). Chapter 11 is concerned with how, in judgment and categorization, the mind can develop different types of representations depending on the information encountered during search, and how these representations can be selected in an ecologically rational way to support good decisions. The inherent limits of people's knowledge imply that a description-experience gap may also exist in other areas of choice. If this is the case, the gap could help to explain puzzling discrepancies that can emerge when lines of research accidentally confound description and experience-such as findings that adults are worse in statistical reasoning than children and babies. Indeed, the limited evidence suggests that the description-experience gap is not a fringe phenomenon limited to monetary gambles. It also appears when people have to identify the causes of an effect based on data that are either described or experienced (Rehder \& Waldmann, 2017), and when people have to reason in a Bayesian fashion about the positive predictive value of a medical diagnostic test (B. Armstrong \& Spaniol, 2017). 
Decision making under risk, categorization, causal reasoning, and Bayesian reasoning are among the mind's major cognitive feats. How well or poorly the mind performs these feats has been taken as a benchmark of human rationality. Given that experiential learning and the accompanying search process may be the most important source of adaptation and a building block of intelligence (Hertwig, Hogarth, \& Lejarraga, 2018; March, 2010), human rationality should not be reduced to performance in tasks from which search and uncertainty have largely been removed. This is also likely to hold in intertemporal choices, where people are explicitly required to make choices about the future. Chapter 9 reviews the critical role that uncertainty plays when people choose between options whose outcomes materialize at different times (e.g., whether to spend their money now or to invest it in the hope of a future payoff). Again, we find that uncertainty and how people search for information can create a description-experience gap and play a defining role in whether people appear to be patient or impulsive in their choices. Furthermore, as chapter 10 demonstrates, this behavioral gap can also be observed in people's risk preferences when they learn, either by description or through experience, about macroeconomic shocks.

\subsection{The Social Mind}

While he was struggling to survive on his desert island, a single data point turned Robinson Crusoe's life upside down: a man's footprint on the shore. "Terrible thoughts racked my imagination about their having found my boat, and that there were people here; and that if so, I should certainly have them come again in greater numbers, and devour me; ... destroy all my corn, carry away all my flock of tame goats, and I should perish at last for mere want" (D. Defoe, 1719/1980, p. 155). Crusoe's terror was well founded. Most new arrivals on the island were man-eating adversaries; their presence posed an imminent threat. Yet his alliance with another human beingFriday, who became Crusoe's close companion-ultimately enabled him to fight off the threat of hostile intruders. Conspecifics are often the most significant aspect of an individual's environment. Humans and other animals compete with others for resources such as food, mates, esteem, or affection; these rivals grant the individual little time for deep thought, protracted information search, or complex calculations. At the same time, they wittingly or unwittingly teach the individual how to deal with a fickle world 
rife with unforeseeable hazards, diseases, and shortages. Social worlds and our knowledge about them present both opportunities and pitfalls (see also chapter 4). In the fourth part of this book, "The Social Mind," we turn to this duality and the challenges it entails.

\subsubsection{Search and the Challenges of Competition}

Animals and humans are constantly faced with important adaptive problems: what to eat, where to live, which mate to choose. Not infrequently, answers must be found under the threat of competition. Under these circumstances, the mind faces different types of uncertainty (e.g., environmental, strategic) and must learn how to balance their consequences. Take, for instance, hermit crabs, which live in the abandoned shells of other sea creatures-usually snails. Since they require larger shells as they grow, they are always on the lookout for a new mobile home. Since the quality of the available shells in the environment varies, a solitary crab faced with merely this kind of environmental uncertainty will thoroughly inspect any potential new home before moving in. However, if several crabs encounter an empty shell at the same time, each individual crab also faces strategic uncertainty, because it does not know the intentions and strategies of its competitors. Under these conditions, what would otherwise be a meticulous exploration is dramatically curtailed (Rotjan, Chabot, \& Lewis, 2010). The crab nearest to the shell will make a split-second decision on whether or not to take it based on a brief visual inspection alone. Once a shell is taken, a chain reaction of shell upgrades may quickly ensue.

This strategic shell game illustrates the challenges and opportunities of sharing the world with others. Exposed to environmental uncertainty alone, the crabs face what is known as the exploration-exploitation tradeoff: whether to continue inspecting a shell (exploration) or to move into it (exploitation). In human choice, the exploration-exploitation trade-off in solitary choice situations has been extensively studied, both theoretically (Brezzi \& Lai, 2002; Gittins, 1979; Gittins, Glazebrook, \& Weber, 2011) and empirically (Cohen, McClure, \& Yu, 2007). The presence of competitors creates an even more complex dilemma. For instance, the more time one takes to explore and thereby reduce environmental uncertainty, the higher the risk that a competitor will act first. There is no perfect escape from this dilemma. Hermit crabs solve it by adjusting their exploration strategies and aspiration levels to the situation at hand, acting as meticulous product 
testers when alone but taking the leap after just a quick peek when faced with fierce competition. Little is known about how people try to come to terms with competition during search. In chapter 12, we examine the extent to which people's search in the external world adapts to the challenges of a competitive environment. Are their search tools as ecologically rational as those of the hermit crab when environmental and strategic uncertainty conspire to create a difficult trade-off?

\subsubsection{Complex Collective Behaviors Often Arise from Simple Rules}

The presence of others does not always mean competition. In many domains, people are better off cooperating than competing. A fascinating domain in which to examine how large groups of people cooperate is individuals' movement through a shared physical space. The motions of many individuals can combine to create complex collective patterns. Consider pedestrian behavior and other self-organization phenomena in crowds. In many cases, such as when unidirectional lanes form spontaneously as people move, collective behavior can be beneficial. In other cases, such as stop-and-go waves and crowd turbulence, collective behavior can be highly detrimental and potentially disastrous, as when people rush to building exits in an emergency. One modeling approach, inspired by Newtonian mechanics, uses models from fluid dynamics and social force theory to conceptualize crowd dynamics (see Moussaïd, Helbing, \& Theraulaz, 2011). This approach treats pedestrians as molecules and their motion as the result of attractive, repulsive, driving, and fluctuating forces, but does not capture the cognitive processes in each mind. Chapter 14 offers an alternative to physics-based models that aims to describe the underlying actual processes: pedestrian heuristics. The view that begins to emerge is that neither strategically demanding interactions (see also chapter 5) nor complex collective behaviors require complex mechanisms (see also Hertwig, Davis, \& Sulloway, 2002). Simple heuristics offer a good starting point for explaining both.

\subsubsection{The Ecological Rationality of the Wisdom of Crowds}

Decision making in humans and animals often occurs in groups. Grouping organisms such as social insects (e.g., ants) must often make rapid decisions about which direction to move in or what action to take in uncertain and dangerous environments. These decisions are rarely solitary. In fact, in 
swarming ants, schooling fish, and flocking birds, effective distributed decision making occurs across a range of environmental contexts (see Couzin, 2009). Pooling information, votes, and preferences in order to make group decisions can be a powerful way of outwitting both the physical and the social environment (e.g., Krause, Ruxton, \& Krause, 2010; Woolley, Chabris, Pentland, Hashmi, \& Malone, 2010). But the wisdom of the crowd is not fail-safe. By all means, tapping the wisdom of others and facing environmental uncertainties together can facilitate solutions that go beyond the capacities of the individual, especially when the problem is difficult to solve alone (e.g., catching larger prey). But collective decisions are not invariably better than individual ones (Sunstein \& Hastie, 2015). Groups can fail to reach better decisions for a number of reasons: for instance, people do not usually become members of a group at random but are selected for specific reasons; selection processes can produce biased groups. More generally, like other decision-making tools, tools for harnessing the wisdom of the crowd are not good or bad per se; their success depends on the problem and the environment at hand. Again, this set of tools can be understood in terms of ecological rationality, as chapter 13 demonstrates.

\subsection{The Unfinished Mind}

The mind's adaptive toolbox is a work in progress. It is never completed. In the fifth part of this book, "The Unfinished Mind," we examine how the life-span trajectory of cognitive development shapes the use of the mind's decision-making tools. Admittedly, little is yet known about the intricate dynamics between cognitive development and the adaptive toolbox, but we can hope to gain glimpses into their interplay (see also chapter 4).

One way we have gained traction on uncovering the dynamics involved is by looking for developmental change that is rooted in the change of core cognitive abilities. Take, for instance, the changes during a person's lifetime in "crystallized" cognitive abilities, such as vocabulary and world knowledge, versus "fluid" abilities, such as reasoning, attention, processing speed, and working memory ( $\mathrm{Li}$, Lindenberger, \& Sikström, 2001). Whereas crystallized abilities increase throughout young adulthood and middle age and then plateau, fluid abilities increase in childhood and adolescence, peak in young adulthood, and decline from middle adulthood through old age. These developmental changes in the mind's cognitive abilities impose 
age-specific constraints for the processes that draw on them. The coupling of these abilities and decision-making tools-as well as their basic building blocks, such as the abilities to activate, represent, maintain, and process information-can be expected to be particularly strong in childhood, when crystallized abilities are least developed, and in old age, when fluid abilities are in decline. It follows that, depending on the cognitive abilities that make up specific decision-making tools, the degree to which these tools are used efficiently will be higher or lower during some periods of cognitive development than others.

By way of illustration, let us return to the recognition heuristic (D. G. Goldstein \& Gigerenzer, 2002). For choices between two alternatives, the recognition heuristic is stated as follows: If one of two objects is recognized and the other is not, then infer that the recognized object has the higher value with respect to the criterion. This heuristic is useful when there is a strong correlation-in either direction-between recognition and the criterion, such as in competitive sports, where successful athletes are more likely to be mentioned in the media and in general conversation than less successful ones and are therefore more likely to be recognized. In contrast, in environments where people or media outlets talk about all objects in question equally often (or equally rarely), recognition is not correlated with the criterion, and the rccognition heuristic should therefore not be used to make inferences. For an ecologically rational use of the recognition heuristic, some knowledge of the environment-particularly of the predictive power of recognition for the target in question-is required. Assuming that world knowledge (crystallized intelligence) grows with age, one might therefore expect that young children use the recognition heuristic less discriminately. This is exactly what Horn, Ruggeri, and Pachur (2016) observed when they investigated the use of the recognition heuristic across individuals in three age groups $(9,12$, and 17 years old). First, they found that elementary school children already made systematic use of the recognition heuristic; second, 9- and 12-year-olds did not adjust their strategy use between domains in which the recognition heuristic resulted in accurate versus inaccurate inferences; third, older adolescents adaptively adjusted their use of the heuristic between domains. These findings suggest that, when the adaptive use of a heuristic requires crystallized abilities (knowledge and experience) and those abilities are still in the making, the heuristic may be used less adaptively. 
The other developmental period during which one may expect a strong connection between cognitive abilities and cognitive tools is old age. During this period, fluid abilities such as working memory are in decline, which can be expected to impact search and learning tools. But what are the consequences? Will aging decision makers explore the world more as their ability to store, extract, and synthesize signals from the sampled data fades? Or will they explore the world less, because their ability to represent, maintain, and process information has declined? These are just a few of the conceptual questions raised by a lifespan perspective on the adaptive toolbox, most of which have barely been investigated. Chapters 16 and 17 report on the progress that we have made in addressing them so far. Chapter 16 deals with the period of adolescence, demonstrating that adolescents respond to uncertainty differently than adults or children and revealing how adolescence is a developmental period fraught with uncertainty. Chapter 17 addresses the development of risk-taking propensity, a major concept in theories of human choice. Two of its main findings are that risk-taking propensity almost universally declines over the adult lifespan and that it is systematically associated not only with the properties and requirements of the decision task but also with environmental conditions such as harsh living conditions.

We also take the analysis of developmental change one step further to include phylogeny. Evolution by natural selection-the longest-running process that produces a fit between environment and behavior-drives developmental change across generations. Chapter 15 demonstrates how computational evolution can be used to examine how natural selection may have shaped the tools in the adaptive toolbox and their ecological rationality.

\subsection{A Systemic View of Uncertainty}

The discussion of uncertainty has a long and winding history, in which scholars from different disciplines have proposed classificatory dichotomies such as that between measurable and immeasurable uncertainties (Knight, 1921/2002) or between epistemic and aleatory uncertainties (Hacking, 1975/2006). The former dichotomy pertains to whether the calculus of probability theory can be called upon to contain uncertainty by quantifying it. The latter concerns the source and cause of uncertainty, namely, lack 
of knowledge or the irreducible random variability of natural processes. Chapter 18 concludes the book with a brief history of the conceptual development of uncertainty and outlines our systemic view of uncertainty. As this view is a leitmotif of this book, we briefly elaborate on it here, returning to the notions of ecological rationality and the adaptive toolbox.

No decision-making tool is inherently good or bad; its success always depends on the structure of the environment in which it is used. So how does an ecologically rational mind decide which heuristic to apply to a specific problem in a specific environment (see also chapters 11,13 , and 15)? If the same decision system were applied to every problem across all environments, this question would not arise. Bayesian statistics, expected utility maximization, and neural networks have all been proposed as such all-purpose, domain-general systems. Due to the flexibility of these optimization models, the fit between the mechanism and the environmental structure is of little concern. In fact, their flexibility makes those that maximize expected utility purely a function of the environment and thus independent of decision makers and the mechanisms upon which they rely (Simon, 1990).

But all general-purpose systems have significant limits (Gigerenzer \& Brighton, 2009). For instance, in highly complex and multidimensional social and nonsocial decision environments, optimization is either impossible or inflicts an unmanageable computational burden. Moreover, optimizing systems do not generalize as well to new situations as do simpler tools. For these reasons, and because simpler decision-making tools offer a realistic theory of decision making, our vision of the mind is not that of an optimizing prediction machine (Clark, 2016). Rather, we think of the mind and its repertoire of competences much as Wimsatt (2007) thinks of nature and evolutionary change, namely, as a "parts dealer and crafty backwoods mechanic, constantly fixing and redesigning old machines and fashioning new ones out of whatever comes easily to hand" (p. 10). From this perspective, the mind-like a good mechanic-learns to select a tool that can address the problem at hand, repurposes existing tools when necessary, and designs new ones using the available evolved capacities of the human mind, such as recognition, emotions, and perspective taking.

The concept of ecological rationality calls for replacing, or at least complementing, the standard dualistic view of uncertainty, according to which the two main sources of uncertainty are human actors with their limited 
knowledge and the environment with its inherent randomness. The necessity of a tool-environment fit for reasonably good decisions means that uncertainty cannot be unambiguously attributed to the actor or the environment, but that it is a property arising from the interactive dynamic of the "married couple" introduced in section 1.3. In this systemic view, uncertainty, as experienced by the individual, is the joint product of environmental unpredictability and the actor's epistemic limitations.

Furthermore, ecological rationality is subject to its own distinct source of uncertainty, namely, that associated with selecting the right tool from the adaptive toolbox for the problem and the environment at hand. Depending on where this uncertainty originates-in the actor, in the environment, or in the actor-environment system-it may represent an "in-between" uncertainty. Resolutions to this uncertainty can be found in the mind (e.g., knowledge of the predictive power of recognition, knowledge about the environment; Pachur \& Hertwig, 2006), in the environment (e.g., time pressure, an environmental structure that precludes any lexicographic ordering of predictors), or in the actor-environment interplay (see the notion of "cognitive niches"; Marewski \& Schooler, 2011). Throughout the chapters of this book, it is this systemic view, implied by the concept of ecological rationality, that guides our investigations.

\subsection{Taming Uncertainty}

As we emphasized at the start of this chapter, every decision that people make involves a kind of prediction: about how much they like something or someone; about where the road less (or more) traveled is likely to lead; about which choices will make them rich or happy. Little is certain, and time pressure, lack of knowledge, the presence of other individuals, limited computational powers, and simultaneous demands on attention do not make things any easier-remember Günter Schabowski. But there is no reason to throw in the towel. The mind, that crafty backwoods mechanic, can draw on a range of boundedly rational tools. It can greatly benefit from simple heuristics because they are so robust across different kinds of social and nonsocial environments. But it is not just heuristics that empower people to sail the seas of uncertainty successfully. Human cognition is about search-collecting new experiences and thereby reducing uncertainty in adaptive ways. Furthermore, individuals can interface with the minds around 
them, harvesting the knowledge and wisdom of others. But in decision making, as in life, there is no such thing as a free lunch. Having many tools means having to choose between them, and this choice can go wrong. The adaptive toolbox is thus both the solution to and the source of uncertainty. If we had to make a prediction, our bet would be that the advantages of the former greatly outweigh the disadvantages of the latter. 